**This is a pre-proofed draft. Final version forthcoming in Evaluative Perception, Ed. A. Bergquist and R. Cowan, Oxford: Oxford University Press**

\title{
Rich Perceptual Content and Aesthetic Properties
}

\author{
Dustin Stokes
}

Both common sense and dominant traditions in art criticism and philosophical aesthetics have it that aesthetic features or properties are perceived. It is common place to hear someone in a gallery say something like "Look how balanced this piece is" or at a symphony performance "Can you hear how unified the string section is?" or at an electronic music show "Can you feel the power of the bass?" These remarks are posed in such a way so as to direct perceptual attention to some aspect of the object or performance. The same is true of criticism. Critical language is rife with examples of aesthetic features being discussed in sensory and phenomenal terms. And one finds the same in philosophical aesthetics, from Baumgarten to Hume to Kant and to more contemporary figures like Budd and Levinson and Lopes. Here is Frank Sibley,

"[A]esthetics deals with a kind of perception. People have to see the grace or unity of a work, hear the plaintiveness or frenzy in the music, notice the gaudiness of colour scheme... They may be struck by these qualities at once, or they may come to perceive them only after repeated viewings, hearings, readings, and with the help of critics" (Sibley: 1965/2001: 34). ${ }^{1}$

One's own phenomenological introspection should suggest the same: if one identifies the somberness of a Wyeth painting or of certain movements of a Mahler symphony, or the frenzy of the Pollock action painting or the Bad Brains punk rock number, does it not seem obvious that these features characterize one's sensory experience? One is most naturally inclined to describe the identified features as part of the phenomenology of one's conscious perceptual experiences. Taken alone, none of these observations are conclusive. But together, the convergence is strongly suggestive: "the aesthetic" is something experienced. Here is a simple thesis that captures the unified thought concerning aesthetic perception:

\footnotetext{
${ }^{1}$ F. Sibley, 'Aesthetic and Non-Aesthetic' Philosophical Review 74 (1965), 135-59; Reprinted in Approach to Aesthetics, Ed. J. Benson, B. Redfern, J.R. Cox (Oxford: Oxford University Press, 2001). See M. Budd, Values of Art, (Penguin Books, 1995); J. Levinson, 'Aesthetic Supervenience', Southern Journal of Philosophy 22 (1984), 93-110 and 'Being Realistic About Aesthetic Properties', The Journal of Aesthetics and Art Criticism 52 (1994), 351-4; D. McIver Lopes, Sight and Sensibility, (Oxford: Oxford University Press, 2005).
} 
AP: Aesthetic properties are sometimes represented by perceptual experience.

The 'sometimes' indicates that aesthetic properties need not be categorically perceptually represented, while it is plausible that they are very often perceptually represented. For all that (AP) has going for it, it turns out there is quite a cast of reasons to be sceptical of the thesis. Here are three.

First, the traditional representationalist model of perception supposes that perceptual experience represents only low-level properties. In the context of art appreciation, one visually perceives the colours and shapes of the painting on the gallery wall, but aesthetic properties like 'being graceful' or 'being vivid', like 'being a mountain' or 'being a dancer' (as represented by the painting), are post-perceptual, represented by a judgment or a belief. So this is a general view about perception and cognitive architecture:

\section{$\mathrm{S}_{\mathrm{c}}$ : Perception represents only low-level properties.}

$\mathrm{S}_{\mathrm{c}}$ implies that AP is false: aesthetic properties are high-level properties, and so are cognized at the level of judgment or evaluation or belief.

The second reason for scepticism regarding AP is ontological. On almost all theories of perception, perceptual experience is descriptive: perception functions to accurately describe or report features of one's environment. Accordingly, if one wants to claim that one sees some aesthetic property $F$, then one thereby commits to the objective, mind-independent reality of Fness. Barring instances of illusion and hallucination, if one is seeing $F$ in some object $o$, then $o$ must really possess feature $F$. And there is a long tradition of worrying about objective aesthetic and moral properties both. So we have:

\section{$\mathrm{S}_{\mathrm{o}}$ : There are no mind-independent aesthetic properties.}

The revision is to say that although one may claim that one sees aesthetic property $F$ in $o$, the 'sees' here should be interpreted cognitively or epistemically: one only judges that $o$ is $F$. 
The third reason is often coupled with this last one. But it centres not on the ontology but the normativity of aesthetic attributions. Aesthetic responses may vary dramatically from subject to subject. Although not the most illuminating characterization, responses to artworks are, as we say, highly subjective. Thus,

$\mathrm{S}_{\mathrm{h}}$ : Aesthetic responses are purely subjective.

This claim is often couched in hedonistic terms: different subjects take pleasure (or not) in different things and experiences. Accordingly, an aesthetic response is something more like a report of how one is struck, pleasurably or not, by an artwork. This implies that matters of taste are not matters of fact. Now, if perception is largely descriptive and veridical, then perceiving aesthetic properties would require representing objective features of the world. But this looks incompatible with the subjective variability of aesthetic response. So, AP must be false and aesthetic assessment a matter of something non-perceptual.

Taken together, these three propositions provide a substantial hurdle for the proponent of AP, and from a variety of theoretical angles: mental architecture, ontology, normativity. They are, however, separable and so AP can largely be defended against each one independently. A substantial amount of work has been done on the second and third reasons for doubt. ${ }^{2}$ Comparatively less has been written on the first challenge, $S_{c}$. For this reason, and in the context of this particular volume of research, the following discussion offers an attempted defence of AP against $\mathrm{S}_{\mathrm{c}}$. If successful, this contributes to vindicating an intuition common to appreciator and theorist alike, namely, that the aesthetic is a perceptual phenomenon.

\section{For and against the sceptical challenge from $S_{c}$}

\section{1.a Sparse versus rich perceptual content}

\footnotetext{
2 There is too much literature to list, so here is just one defence of AP for each of the other two sceptical worries. On the second, ontological, reason for doubt, Frank Sibley (1968) argues by analogy that aesthetic attribution meets (enough of) the same standards as colour property attribution. And insofar as meeting these standards suffices for some kind of objectivity (not necessarily "purely" mind-independent objectivity), then aesthetic qualities may be properly understood as perceivable, genuine properties. See F. Sibley, 'Objectivity and Aesthetics', Proceedings of the Aristotelian Society, Supp. Vol. 42 (1968); Reprinted in Approach to Aesthetics, Ed. by J. Benson, B. Redfern, J.R. Cox (Oxford: Oxford University Press, 2001). Regarding the third, hedonic, reason for doubt, David Hume's (1757) solution to the problem of taste remains one of the best. Aesthetic experience is, for Hume, grounded in perceptual experience. And although different subjects respond differently to the same work, there are a number of ways that perception can be deficient. Objectivity in this context is thus revealed by identification of an ideal judge who enjoys unhindered perceptual experience of works. So although subjective, one's aesthetic responses are more or less appropriate in a way that depends upon how well one's perceptual experience of works approximates that of the ideal judge. See D. Hume, 'Of the standard of taste,' The Philosophical Works of David Hume, Ed. by T. H. Green and T. H. Grose. 4 volumes (London: Longman, Green, 1874-75).
} 
From a lay perspective, we do not often distinguish the features that "just appear" to us from those that require some interpretation or judgment. But cognitive science has made a tradition of attempting to do just that, and one dominant view is conservative with respect to those features that simply appear (to all normal perceivers), while a lot more is admitted into the contents of interpretation, judgment, and belief. So as you look out your window, vision carries information about the colours and shapes of the afternoon sky. You see blues, yellows, whites, and greys, and the outlines of the clouds and distant mountain range. But representation that an object is of a natural or artefactual kind is done at the level of cognition. So you judge the presence of the mountain range or of the clouds. These properties—being a mountain, being a cloud—while mentally represented by you, are not represented by vision.

It is worth taking a brief moment to clarify some motivation for the traditional, sparse view. Although it takes some time to develop beyond the Jamesian blooming, buzzing confusion of sensory stimuli, the vast majority of human beings develop normal perceptual capacities, essential for making sense of (literally) and acting upon objects and events in the immediate environment. And for each sense modality, there is a dominant set of norms, a convergence on colour discrimination, tone discrimination, and so on. Add to this the apparent fact that once developed, perceptual systems work extremely quickly, with no person-level effort (once proprietary input has been received by the relevant receptors), and in a way that most typically accurately represents the immediate environment. This has encouraged many theorists to think that perceptual systems must be biologically hard-wired, and must process limited, modalityspecific classes of information. Put one way, input from other non-sensory parts of the cognitive system (what one knows, one's goals, and so on) would undermine the observed cross-perceiver convergence on discrimination, speed, and objectivity and so, by inference to the best explanation, perceptual systems must function largely independently of those cognitive systems, processing only basic, context-neutral features of the environment.

Generalizing, we have the claim that grounds the relevant sceptical challenge to AP:

\section{$\mathrm{S}_{\mathrm{c}}$ : Perception represents only low-level properties.}

The incompatibility approaches logical contrariness: If perception represents only low-level properties, and all theorists agree that aesthetic properties are not low-level properties (if "real" 
properties at all), then AP is false: aesthetic properties are never represented by perceptual experience. $^{3}$

\section{1.b: Two aesthetic cases and two categories of explanation}

Being dynamic and being serene are standard examples of aesthetic properties. ${ }^{4}$ They also typically oppose one another: it is rare that one finds an object to be both dynamic and serene. Now consider the following thought experiment, modeled on a famous example given by Kendall Walton. First, suppose you are in Madrid and you visit the Museo Nacional Centro de Arte Reina Sofía, which houses Picasso's Guernica. As most readers would agree, experience of Guernica, in this context, is well-described as dynamic, violent, disturbing, vital. Now imagine that, on a different occasion, you visit the Gallery of Guernicas. This is the gallery of Walton's hypothetical society of artmakers who produce works of art in the category of guernicas. Works in this category are "like versions of Picasso's Guernica done in various bas-relief dimensions. All of them surfaces with colors and shapes of Picasso's Guernica, but the surfaces molded to protrude from the wall like relief maps of different kinds of terrain. Some guernicas have rolling surfaces, others are sharp and jagged, still others contain several relatively flat planes at various angles to each other, and so forth" (Walton 1970: 347). ${ }^{5}$ Now there is no actual category of guernicas for reference, so the reader must simply imagine the perceptible contrast between Guernica-makers' guernicas and Picasso's Guernica. And then imagine that in the Gallery of Guernicas, after many viewings of the Guernica-makers' works, you turn the corner and encounter Picasso's Guernica. It is very likely that in this rather different viewing situation you would describe your experience of Picasso's work as cold, or lifeless, or serene, or "perhaps bland, dull, boring-but in any case not violent, dynamic, and vital” (Walton 1970: 347). ${ }^{6}$ To select a property each from Walton's lists, in

\footnotetext{
${ }^{3}$ There is no standard definition of 'low-level' or 'high-level' as pertains to perceived properties, but instead a standard contrast. Susanna Siegel characterizes high-level properties negatively, where high-level properties for vision are those "other than color, shape, illumination, motion, and their co-instantiation in objects" (2006: 481) See S. Siegel 'Which Properties Are Represented in Perception?', in Perceptual Experience, Ed. by T.S. Gendler and J. Hawthorne (Oxford: Oxford University Press, 2006). The discussion here will just follow this convention. Likewise 'sparse content' will characterize experiences, as theorized, that involve representation of only those low-level properties; 'rich content' will characterize experiences, as theorized, that involve representation of something more than those basic, or low-level properties.

4 "Standard" at least according to one common picture given in analytic aesthetics. See F. Sibley, "Aesthetic Concepts', Philosophical Review 68 (1959), 421-50. There is of course substantial debate regarding what makes a property (or feature or concept or term) aesthetic vs. non-aesthetic. See F. Sibley, 'Aesthetic and Non-aesthetic', Philosophical Review 74 (1965), 135-59; T. Cohen, 'Aesthetics/Non-Aesthetics and the Concept of Taste,' Theoria 39 (1973), 113-52; P. Kivy, 'What Makes “Aesthetic” Terms Aesthetic?', Philosophy and Phenomenological Research 36 (1975), 197-211. Here it is just assumed that there is some, perhaps loosely delineated, set of paradigm aesthetic properties.

${ }^{5}$ K. Walton, 'Categories of Art', Philosophical Review 79 (1970), 334-67.

${ }^{6}$ One way to think of this is in Walton's terms of categories of art. According to Walton, works can be perceived under different categories of art and, accordingly, the aesthetic properties we are likely to attribute can vary, and sometimes quite dramatically. And since one may know more or less about art, and thus more or less about artistic
} 
the first case one attributes being dynamic to Guernica; in the second case, one attributes being serene to the same work, Guernica. Take this as our first case of a contrast in aesthetic reaction.

Consider a second case. Most of us are familiar with Impressionist paintings. But of course each of us had to learn about this particular movement (and learn by viewing, either in person or through reproductions). Once learned, it is plausible that one thereby learns to identify, by sight, impressionist paintings as such (with varying levels of precision and reliability of course). So at one point, perhaps as a child, one did not have the capacity to attribute 'impressionist' to paintings with any reliability and, at a later point, one acquired this capacity and then readily and accurately identifies paintings in just this way. A natural description of the latter says that some paintings just strike one as impressionist. Furthermore, it is natural to describe impressionism in terms of a gestalt, even if that gestalt cannot be defined in rigorous terms. Impressionist paintings are typified by a number of perceptible features: highlighting of natural light and reflection; a regular (but not categorical) use of lighter colours; identifiable quick, short strokes of paint; an emphasis on a scene rather than any one figure or group of figures; use of angles and composition creating a candid rather than posed depiction of people and events. To know impressionism, is to know and respond to some cluster of these features. And this is an important difference in the aesthetic reaction of the naive vs. experienced viewer. ${ }^{7}$

Summarizing, these are both cases of contrast: one where one subject responds to an artwork (or category of artwork) in one way, and a distinct subject (or the same subject in a different context) responds to the same artwork in a clearly different way. This difference in aesthetic reaction entails a difference in the overall mental experience of the two types of subjects. Plausibly, this difference will involve a difference in the overall feel or phenomenology of the contextually distinct viewings (in the first case) and the naive viewing vs. the informed viewing (in the second case). ${ }^{8}$ And by hypothesis, this difference depends upon some difference in learning, experience,

categories (e.g. one may only know the category PAINTING but not the category ABSTRACT EXPRESSIONISM), one's aesthetic attributions can vary with one's knowledge about art. In the present example, in the ordinary case, one would perceive Guernica under the category PAINTING or maybe CUBISM; but in the hypothetical case, one perceives Guernica under a very different category, GUERNICA. Importantly, the perceptible features that make a difference to aesthetic reaction can vary from category to category, such that reaction to the very same work can vary as a result of a change in the category (or categories) that a subject perceives them in. The question for present purposes is whether this contrast in aesthetic reaction is a genuine perceptual one. See Op. Cit. note 6.

7 One might maintain that 'being impressionist' is an artistic property, but not an aesthetic property, perhaps because it seems to be a property instantiated only by artworks, by contrast to more broadly-instantiated properties like 'being dynamic' or 'being balanced'. Granting this distinction makes no difference to the argument that follows. 8 This is not meant to be question-begging in the current dialectical context: the overall phenomenology of one's mental experience may include more than just sensory phenomenology. See Siegel 2006, Op. cit. note 3. 
or knowledge. The question is how this difference should be further explained and whether the most plausible explanation favours $\mathrm{S}_{\mathrm{c}}$ or AP?

Two categories of explanation are relevant. The first explanation says that the aesthetic difference is explained post-perceptually: the informed viewer makes different judgments or evaluations, premised on the knowledge she has and the naive lack. But this is not, and does not depend upon, a perceptual difference. ${ }^{9}$ Put in its strongest form, the perceptual experiences of the distinct subjects are the same (in phenomenal character and representational content). If pressed, this theorist could concede a sensory phenomenal difference, but this difference would be explained in terms of sparse content only.

The motivation is this: if aesthetic properties like 'being dynamic' and 'being impressionist' are high-level properties, then they are not represented by experience. Again, perception is fast, hardwired, and objective across perceivers and cultures, and so plausibly only represents basic properties of the environment. An explanation of this kind is further motivated by acknowledgement of the (hypothesized) fact that the naive versus informed subjects differ in what they have learned about artworks and aesthetic features. So, the reasoning would go, the resultant (aesthetic) differences will be at the level of post-perceptual cognition. One learns some typifying features of impressionism and is then able to judge and report which paintings are impressionist. And note finally how these two points work in tandem: if one is already committed to the claim that perception represents only low-level properties, and the difference (between naive and informed subjects) involves high-level properties the identification of which depends on background learning, then it must follow that those properties will (post-learning) be represented in later cognitive (non-perceptual) processes.

Now for the second category of explanation. Consider once more our two examples. In each case, there is a difference in the aesthetic reaction of the two subjects that depends upon some background learning or cognition. And plausibly, the overall experience between naive and informed subject will differ in broad phenomenology. How can the proponent of AP explain these differences?

\footnotetext{
9 A great deal more could be said about candidate cognitive states or processes here. For example, is there a difference between a judgment and an evaluation? What is the role of (occurrent) belief? These questions should be analyzed, but not here. All that matters here is that there are a number of cognitive states typically theorized and, their possible differences notwithstanding, they are all supposed to be non-sensory, post-perceptual states. Accordingly, this is a category of explanation unified by its distinction from the second category involving perceptual explanation.
} 
The general type of explanation says that the phenomenal contrast is best explained as a difference in rich perceptual representation. There is a line of argument now standard in the literature on admissible contents that appeals to phenomenology. Susanna Siegel argues that the overall phenomenal difference before and after one learns to recognize pine trees is a perceptual difference. For the pine tree-spotter, vision represents pine trees; and for the pine tree-naive, it does not. ${ }^{10}$ This position has been buttressed with some empirical support. For example, Tim Bayne argues that the difference between the visual associative agnosic and a normal human perceiver is that while the former possesses intact low-level perceptual capacities, she lacks intact high-level perceptual capacities. ${ }^{11}$ So the agnosic sees the telephone (its spatial and colour properties) but does not visually recognize the telephone. Bayne thus draws an inference on the basis of what's missing for the agnosic: normal human perception represents high-level properties like 'being a telephone'. One can see how this line of argument provides a template for thinking about rich perceptual representation of aesthetic properties. ${ }^{12}$

In the discussion that follows, a new argument for rich perceptual representation is offered. This argument employs Siegel's contrast method, as well as some of her original analysis, but focuses on how to best understand mental occurrences described as 'seeing-as', with an emphasis on the phenomenology of visual perception of ambiguous figures. It takes inspiration from related discussions in Wittgenstein and N.R. Hanson. ${ }^{13}$ The results of this argument are then applied to the cases of aesthetic contrast, where the aesthetic cases are specially illuminating. This general explanation can then be extended by appeal to three different kinds of mental mechanism. This abundance of explanatory options for the proponent of AP shifts the burden of proof to the critic of AP.

\section{Ambiguous figures and the argument from seeing-as}

\section{2.a The argument}

\footnotetext{
10 See Op. cit. note 3 and S. Siegel, The Contents of Visual Perception (Oxford: Oxford University Press, 2010). ${ }^{11}$ See T. Bayne, 'Perception and the Reach of Phenomenal Content,' Philosophical Quarterly 59 (2009), 385-404. One can find similar arguments, or at least suggestions, in R. Van Gulick, 'Deficit Studies and the Function of Phenomenal Consciousness', in Philosophical Psychopathology, (Cambridge, MA: MIT Press, 1994, 25-49) and C. Siewert, The Significance of Consciousness (Princeton, NJ: Princeton University Press).

${ }^{12}$ For a discussion of this sort, see D. Stokes, 'Cognitive Penetration and the Perception of Art', Dialectica 68 (2014), 1-34.

13 See L. Wittgenstein, Philosophical Investigations, Trans. by G.E.M. Anscombe (1953), 4th Edition Ed. by P.M.S. Hacker and J. Schulte (London: Wiley-Blackwell, 2009); N.R. Hanson, Patterns of Discovery, (Cambridge: Cambridge University Press, 1958) and Perception and Discovery: An Introduction to Scientific Inquiry, (San Francisco: Freeman and Cooper, 1969).
} 
Figure 1 includes two famous examples of ambiguous figures: the duck/rabbit and the Rubin goblet. ${ }^{14}$ Consider the duck/rabbit. First see it as a duck. Then see it as a rabbit. The term 'seeing-as' comes naturally here. ${ }^{15}$ The same goes for the Rubin goblet. First see it as a goblet. Then see it as a pair of faces directed at one another. Here again, you see as a goblet or see as a pair of faces (and cannot do both simultaneously). Plausibly, the overall experiences of seeing the image as a duck versus seeing the image as a rabbit differ in phenomenology: what it's like to see something as a duck is broadly different from what it's like to see it as a rabbit. The most natural explanation of these observations is that the switch from seeing-as a duck to seeing-as a rabbit involves a change in visual representation. The difference is a genuine perceptual difference. Same goes for the Rubin goblet and many other ambiguous figures.

How might a proponent of $S_{c}$ explain these switches while denying any rich perceptual difference? Here the low-level theorist will encounter a challenge in the form of a trilemma. First, a low-level theorist could claim that there is no phenomenal difference between seeing-as a duck and seeing-as a rabbit. Second, one could grant that difference but claim that it is an entirely cognitive phenomenal difference. Third, one could claim that there is a difference in judgment accompanied by a change in low-level perception only. Each of these explanations is either less plausible than the opposing high-level perceptual explanation, or collapses into that very explanation.

The first horn has the low-level theorist rejecting the claim that there is a difference in the phenomenology of seeing-as a duck versus seeing-as a rabbit. There is no difference in what it's like to have these two experiences. This position is implausible. A theory that denies that it feels different to see an image as a duck versus see it as a rabbit is almost entirely lacking in intuitive force, looking instead like an unfortunate theoretical consequence. Intuition and introspection are fallible, but both strongly favour phenomenal differences between the two experiences. ${ }^{16}$

\footnotetext{
14 The reader should note the particular duck/rabbit image used. The choice of this image over the also common, mere two-dimensional outline shape image is deliberate, since the latter may not work for the line of reasoning that follows, and for reasons that hopefully become clear.

15 Indeed, for whatever it is worth, it is incredibly difficult to write these instructions in some non 'seeing' or "perceiving'-involving way. "Judge it to be a duck", "Interpret it as a duck", "Cognize it as a duck", "Believe it is a duck"... are all forced at best.

16 This does not seem to be the sort of claim for which further argument is appropriate, since it flatly appeals to intuition. That said, and for what it's worth, a number of disparate theorists share the intuition, as discussed in Macpherson 2006. See F. Macpherson 'Ambiguous Figures and the Content of Experience', Noûs 40 (2006), 82-117; R. L. Gregory, The Intelligent Eye (London: Weidenfeld \& Nicholson, 1970); A. Millar, Reasons and Experience, (Oxford: Oxford University Press, 1991); M. Tye, Ten Problems of Consciousness: A Representational Theory of the Phenomenal Mind (Cambridge, MA: MIT Press, 1995).
} 
Second horn: a low-level theorist may grant the difference in phenomenology, but explain it as deriving from a difference in the non-perceptual cognizing of the two images. This claim is also problematic. First, cognitive phenomenology (by contrast to sensory phenomenology) is controversial. Many have argued that there is nothing it is like to have a belief or make a judgment. ${ }^{17}$ More substantively and less theory-committal, examples where judgment and phenomenology come apart are easy to come by. First, there are cases where a change in judgment causes no change in phenomenology; visual illusions illustrate this point. Most are familiar with the Muller-Lyer illusion (Figure 2), but of course each of us learned at some point that it is an illusion. It is commonly accepted that as one's beliefs (or judgments) about the lengths of the lines change (one learns that the two lines are in fact of the same length), one's phenomenology stays the same. The illusion persists and there is no obvious difference in what it is like across the pairs of experience. ${ }^{18}$ Second, there are many cases where phenomenology changes while judgment remains the same. Consider colour constancy. Imagine a pink book on your desk, illuminated by the setting sun, moving from bright sunlight at $\mathrm{t}_{1}$ to the last sunlight of the day at $t_{2}$. Across this entire window of time, your belief that the book is pink is stable. And, in one sense, you still perceive the book as pink (per constancy mechanisms) and would judge it accordingly. However, it is undeniable that there is a difference in what it's like to see the book at $\mathrm{t}_{1}$ versus what it's like to see the book at $\mathrm{t}_{2} \cdot{ }^{19}$ So, phenomenology comes apart from cognition (judgment) in both directions. The theorist under consideration would have to claim,

\footnotetext{
17 One classic critic of cognitive phenomenology is D. Dennett, 'Quining Qualia', in Consciousness in Modern Science, Ed. by A. Marcel and E. Bisiach, (Oxford: Oxford University Press, 1988). See also J. Kim, Physicalism, or Something Near Enough, (Princeton, NJ: Princeton University Press, 2005). For arguments that cognitive states enjoy distinctive phenomenology, see Siewert 1998, Op. cit. note 13; O. Flanagan, The Science of the Mind, 2nd edn (Cambridge, MA: MIT Press, 1991); T. Horgan and J. Tienson 'The Intentionality of Phenomenology and the Phenomenology of Intentionality,' in Philosophy of Mind: Classical and Contemporary Readings, Ed. by D. Chalmers, (Oxford: Oxford University Press, 2002); B. Loar, 'Phenomenal Intentionality as the Basis of Mental Content', in Reflections and Replies: Essays on Tyler Burge, Ed. by M. Hahn and B. Ramberg (Cambridge, MA: MIT Press, 2003). For a recent volume of papers on this topic, see T. Bayne and M. Montague, Cognitive Phenomenology, (Oxford: Oxford University Press, 2012).

18 Macpherson 2006 makes this point, partly in service of a challenge to nonconceptual intentionalist theories of perception who, she argues, struggle to explain experiences of ambiguous figures, once it is granted that the gestalt shifts involve shifts in phenomenal character. The discussion of ambiguous figures in the present paper is indebted to Macpherson's analysis. See Macpherson, Op. cit. note 17.

19 Some will note that constancy phenomena may, for reasons intimated here, be taken as a challenge to intentionalist theories of perception, since it appears that we have an instance where representational content is the same across phenomenal changes. An early example of this challenge can be found in C. Peacocke, Sense and Content: Experience, Thought, and Their Relation, (Oxford: Oxford University Press, 1983). More recently, see B. Millar, 'Colour Constancy and Fregean Representationalism,' Philosophical Studies 164 (20134), 219-31. For a representationalist defence, see F. Dretske, 'Experience as Representation,' Philosophical Issues 13 (2003), 67-82. And for alleged explanations of constancy phenomena in terms of Fregean content, see D. Chalmers, 'Perception and the fall from Eden,' in Perceptual Experience, Ed. T.S. Gendler and J. Hawthorne, (Oxford: Oxford University Press, 2006) and B. Thompson, 'Senses for Senses,' Australasian Journal of Philosophy 87 (2009), 99-117. For general discussion, see S. Siegel, 'The Contents of Perception,' The Stanford Encyclopedia of Philosophy,' (2010), http://plato.stanford.edu/archives/win2014/entries/perception-contents/ .
} 
implausibly, that all cases of seeing-as are somehow different, where changes in relevant judgment (that is a duck) always come with changes in non-sensory phenomenology.

The intermediate conclusion to this point is that seeing-as is most plausibly understood as a sensory perceptual phenomenon. For some object $o$, seeing $o$ as an $F$ is a distinct perceptual experience from seeing $o$ as a $G .^{20}$ The final step is to extend the argument to defend a rich perceptual content thesis. This third horn of the trilemma requires more lengthy discussion.

First consider the ambiguous figures just discussed. It is eminently plausible that (assuming a non-naive viewer), when one sees the duck/rabbit figure, say, as a duck, some of a cluster of properties - 'being a duck' or 'being a duck appearance' or 'being an image of a duck' or 'being like a duck' - is thereby mentally represented as instantiated. ${ }^{21}$ And note that each of the properties in this disjunction is a clear example of a high-level property. If this is correct, and the first two horns of the trilemma are successfully challenging, then there remains one way for the low-level theorist to maintain her position. What this theorist must say is that the phenomenal difference (from switch to switch) is at the level of low-level property representation, while 'being a duck' and the other relevant high-level properties are cognitive contents (e.g. one interprets the image as a duck, or as a duck representation, or as being a duck appearance, and so on). The question is what the former difference in perceptual content amounts to. It is both introspectively and psychologically implausible that each time the switch occurs, perception represents distinct colours or distinct edges (with the same duck/rabbit figure in view throughout). Moreover, as again introspection should reveal, it isn't just (or even) the outline shape that changes. Instead, such a change would have to be in something like an overall gestalt, some organization of low-level properties. A familiar way to put this is that the duck (or the rabbit) "pops out". So, for the duck/rabbit image, when one sees it as a duck, call the candidate, pop-out feature of content 'being organized like a duck'. Is it correct to insist that this kind of

\footnotetext{
${ }^{20}$ This should be qualified with 'sometimes' or 'for many Fs and many Gs', since this is not intended as, nor need it be, a categorical claim about all instances of seeing-as. For example, in cases of indiscernibles-say a pair of identical twins - there may be no perceptual, phenomenal difference between seeing the same object as being one or the other of the indiscernible pair (for instance, seeing an individual as being one or the other twin). The argument here only needs to be plausible for many, but not all, uses of 'seeing-as'. And furthermore, there may be deviation in, or different uses of, 'seeing-as'.

${ }^{21}$ This qualification is made for a pair of reasons. First, there are extra complications introduced since, strictly speaking, the duck/rabbit figure is a mere depiction and so one does not take there to be (in any sense of mental representation) a real, living, breathing duck in one's visual field (when viewing the duck/rabbit figure). Second, there is an open question, not sufficiently discussed in the literature, about whether the rich content theorist needs to claim that kind properties (e.g. the natural kind property 'being a duck') are themselves part of the content of perceptual experience. It is plausible, as discussed momentarily, that a much weaker claim is sufficient for rich perceptual content.
} 
perceptual representation of an organization of low-level features involves only low-level content?

This is a fine line to walk. Note that the switches in question are reliable, in the sense that (with these and most ambiguous figures) one switches between only two things: now it's a duck, now it's a rabbit, now it's a duck, etc, and one will tend to have the organized-like-a-duck experience only when appropriate stimuli are available (one will not have that kind of experience when viewing, say, the Rubin goblet or a sunset). Furthermore, the phenomenology is, all else being equal, relatively stable across these switches: it isn't as if the figure strikes the perceiver, across a range of switches, with a wide variety of duck-appearances. ${ }^{22}$ All of this is supposed to be explained by the low-level theorist under consideration as a switch in interpretation (between 'that's a duck' and 'that's a rabbit') and an accordant shift between visual experience representing the world as containing the relevant organizational gestalt (as containing an overall organization of edge, colour, and shape typical of a duck, then of a rabbit, etc). These considerations in hand, what reason is left to deny that this content is rich content of visual experience?

One way to put things is in terms of discriminatory capacities. The low-level theorist in question says that one's visual experience characterizes some cluster of low-level properties (colours, edge, shapes) as being 'organized like a duck', where this is the (or at least a) appearance-a coherent organization of looks - typical of a duck. And she must grant further that tokens of this visual experience type reliably co-vary with ducks or duck images or duck appearing objects. This implies a visual capacity for discriminating a kind of thing: things whose basic features are organized so as to appear like a duck. And this is just another way to say that vision discriminates — that is, represents—more than just colours, shapes, edges, motion, and location. It represents objects in the world as organized, where those organizational gestalts correspond in some systematic way to the kinds of objects that populate one's environment. This perceptual discrimination manifests in instances where we recognize an object (or image) as being of this

\footnotetext{
${ }^{22}$ In this way, at least when applied to ambiguous figures, this proposal is distinct from the one criticized in Siegel (2006, Op. cit. note 3), in her defence of perceptual representation of kinds.. One rejoinder from the low-level theorist she considers is that, in the case of pine tree spotting, one perceptually represents a general pine-tree gestalt that is invariant across differences between individual pine trees. Siegel argues, plausibly, that this is phenomenologically implausible, considering at least a series of viewing distinct pine trees. But the suggestion here is that two distinct overall organizational gestalts, and fairly stable ones, are likely to correspond, phenomenologically, to the switch between seeing-as a duck and seeing-as a rabbit. And in this case, unlike the pine tree case, there is not a variety of distinct stimuli (since one is just viewing the same duck/rabbit figure). Generalizing, the suggestion here is not that, when seeing-as, we token some stored, individual-invariant gestalt representation. This is what Siegel seems to have in mind, but surely this is not the only way to think about perceptual organization vis-a-vis "gestalts".
} 
kind or that kind, or at least, recognize an object as appearing as being of this kind or that kind. We see the image as a duck and then as a rabbit. ${ }^{23}$

Now recall the two aesthetic cases. In the first case, a perceiver, in two importantly distinct contexts of appreciation, ascribes first 'dynamic' and then 'serene' to Picasso's Guernica. The second case contrasts a subject with the capacity for attributing 'impressionist' to works with a subject who lacks this capacity. In both cases, there is a contrast in aesthetic reaction. Here again, it is natural to describe the contrast as a perceptual one: one sees the Guernica as dynamic or sees it as serene. One sees a work as impressionist or not. An opponent of AP who wants to maintain that this is either a purely cognitive difference or a difference only in low-level perception encounters the same trilemma.

The first and second horns of the trilemma are applied here as they were to ambiguous figures. First, a claim that there is no phenomenological difference between aesthetic reactions is implausible. What it is like to react to an artwork as being dynamic is phenomenally distinct from what it is like to react to the work as serene. Second, the claim that all phenomenal differences are purely cognitive is implausible, saddling the objector with rich cognitive phenomenology, and contrary to common instances where judgment (or other cognitive states) and phenomenally characterized experience come apart. These two horns are sharpened in the aesthetics cases. Both ordinary opinion and ordinary language suggest that aesthetic reactions to artworks are sensory in character. Anyone unversed in sophisticated philosophical debate about these topics would default to a sensory-phenomenal characterization of recognizing the dynamism or impressionism in a work. Same goes for both artists and art critics. Visual artists, for example, create works with intentions of establishing certain aesthetic 'looks' or 'appearances', and assume that their uptake is visual. And critical discourse is full of aesthetic description couched in sensory terms. ${ }^{24}$

The final horn challenges the theorist who grants a phenomenal change, but maintains that it is a feature only of low-level perception. So, the difference between the impressionist-spotter and the impressionist-naive is that the first, and not the second, judges of a work 'that is impressionist', and this is accompanied by some difference in low-level perceptual representation. At least in

\footnotetext{
${ }^{23}$ Bear in mind that the point is not one about linguistic representation. Figuring in the content of perception does not require some tokening of a linguistic symbol. This is equally true for the property 'being red' as it is for the property 'being a duck'; it is not a condition on perceptual content that 'red' or 'duck' is mentally represented.

${ }^{24}$ See also Stokes 2014 for discussion of these matters, and in relation to the cognitive penetrability of perception. Op. cit. note 13 .
} 
this type of case, it is implausible that there is a difference in basic colour or shape or edge perception. More plausibly, there is some difference in the way these basic features are perceptually organized. The impressionist spotter enjoys perceptual experience as of an impressionist gestalt. And here, as with ambiguous figures, the most plausible way to understand this option is in a way that concedes rich perceptual representation. And with the aesthetic case, once again, things can be sharpened. Unlike natural and artefactual kind properties, which on many accounts have a non-perceptible essence or kind-determining underlying structure, many aesthetic properties are exhausted by appearance features. ${ }^{25}$ There is nothing to the property of 'being dynamic' or 'being impressionist' (at least when in the context of aesthetics and artworks) beyond an organization or gestalt of basic features. ${ }^{26}$ Accordingly, if a work is recognized as 'being impressionist', and perceptually recognized as an overall organization, then 'being impressionist' is perceptually represented. Therefore, in the aesthetic case, if the low-level theorist (or other sceptic of AP) grants a sensory difference in terms of the perceptual organization of features, this is equivalent to granting that aesthetic properties are perceived. And to grant this is to grant that some high-level properties figure in the content of experience.

A clarification of the argument at this stage will be useful. The argument is not, simply, that because (many) aesthetic properties are appearance properties, they must be perceptually represented (if mentally represented at all). Instead, considering pairs of contrasting aesthetic reactions, attempts to explain the contrast in strictly non-phenomenal ways (Horn 1) or strictly cognitive ways (Horn 2) are implausible. The opponent of AP is then left with a hybrid explanation, where a contrast in judgment is accompanied by distinctive sensory phenomenology (Horn 3). The latter perceptual differences are not sufficiently explained in terms of only colour or shape or edge perception, but instead require some appeal to an overall organization of those basic features. But, to perceptually experience that kind of organization-an impressionist gestalt-just is to perceptually experience the relevant aesthetic property. Therefore, aesthetic

\footnotetext{
25 The 'many' qualification here is deliberate. As already noted, just what is and is not an aesthetic property (or quality, term, or concept) is controversial. And one point of debate is whether aesthetic properties are, or are exhausted by, appearance properties. For example, it is plausible that literary works have aesthetic qualities, but at least some of those qualities have nothing to do with the visual or auditory appearance of the words as they appear on the page or as they are spoken. This general debate can be reasonably avoided, since the case being made here for rich perceptual content of aesthetic properties only requires that some aesthetic properties are exhausted by appearance. The discussion will assume this qualification, even if it is not always made explicit with 'some' or 'many'. ${ }^{26}$ Note that the question about the relation between aesthetic properties and basic properties is important, but orthogonal to the present line of reasoning. Note also that this is not to suggest that there is no objectivity to aesthetic properties or that there are no correctness conditions for aesthetic responses and attributions.
} 
reaction sometimes involves perceptual representation of high-level properties. It involves rich perceptual content. ${ }^{27}$

\section{2.b A rejoinder and some general lessons}

The low-level theorist might reply to the above argument by taking on Horn 3, as follows. Grant that these instances of seeing-as involve some rich content, while maintaining that genuine kind properties are not themselves perceptual contents. One way to articulate this rejoinder is in familiar terms of accuracy conditions. If perceptual experience is representational then, in a rather intuitive sense, it says to the perceiver how the world is here and now. ${ }^{28}$ Representations can be more or less accurate with respect to how they represent; perceptual experiences can be more or less accurate in what they say about how the world is here and now. Accordingly, for any experience token $e$, one can identify the way(s) $w$ the world would need to be (here and now) for $e$ to be accurate (setting to one side difficulties about degree of accuracy). If the world is way $w$, then $e$ is accurate (and if not, then not). To specify these accuracy conditions is to specify what any experience $e$ is about; it is to specify the content of $e$. The debate about admissible contents concerns the types of properties that are needed in order to specify accuracy conditions and, thereby, to adequately capture the content of perceptual experience. Here two questions should be distinguished: (1) Are basic properties sufficient (so, for vision: colour, edges, shape, motion, location)? (2) Are natural or artefactual kind properties needed? It is often assumed that if one answers 'no' to question (1) then one answers 'yes' (perhaps must answer 'yes') to question (2). Working through this final rejoinder reveals that this may be an unnecessarily coarse way to think about the richness of perceptual representation.

Consider once more the ambiguous figures. The option being considered (Horn 3) is that seeingas a duck in the presence of the duck/rabbit image involves a judgment that 'that's a duck' plus a perceptual experience that is best characterized as 'being organized like a duck'. The low-level theorist might attempt to maintain that the content of this perceptual experience is low-level by reasoning as follows. Plausibly, one will have this experience in a variety of circumstances, only some of which involve the instantiation of the property 'being a duck'. Thus, one will token this (broadly individuated) experience type in the presence of living, breathing ducks, or in the presence of plastic duck decoys, or certain landscape paintings or, as in the case in question, in

\footnotetext{
${ }^{27}$ This discussion owes much to Siewert's 1998 discussion of perceptual recognition and organization, Op. cit. note 12.

${ }^{28}$ Siegel 2010 argues that a rich content thesis could be suitably adjusted even if a content thesis fails, for example, if one favours some direct realist theory of perception. Op. cit. note 11, 97.
} 
the presence of the duck/rabbit image in Figure 1. Furthermore, it seems plausible that the experience is accurate if one is in the presence of any of these stimuli. And notice that the natural kind property 'being a duck' is instantiated only in the first circumstance listed here. So it cannot be the case that the kind property 'being a duck' is part of the content of the relevant experience, since it only figures (and need only figure) as one factor in a disjunctive set of accuracy conditions. In other words, there is an array of circumstances, any one of which would render the experience accurate but only one of which involves instantiation of the relevant kind property. By contrast, the various low-level properties are part of the content of the experience. So, if part of the character of the experience involves a vertical line or edge (say part of the duck-outline), then this experience (or sub-experience, if one prefers) is accurate only if there is a vertical line or edge in one's presence. The basic, low-level property 'being a line (with such-and-such orientation)' must be instantiated here and now. Accordingly, this low-level property is part of the content of the experience. Notice further how this will be part of the content no matter which of the listed circumstances obtain (real duck, duck decoy, duck/rabbit image, etc.). So the low-level theorist argues by disanalogy, and concludes that while the relevant experience involves the 'being organized like a duck' character, that character is sufficiently captured by appeal to basic properties only. In particular, the property 'being a duck' is unnecessary in specifying the representational content of the experience. And the disanalogy generalizes, low-level properties are necessary to specify perceptual content (as accuracy conditions) while kind properties are not necessary to specify content.

There is an important insight in this rejoinder, but it will not service the low-level theorist's defence. The argument was that kind properties are unnecessary for specification of content (as accuracy conditions), and by an alleged disanalogy with perceptual representation of basic features like colours and edges. The theorist is right about kind properties, but wrong about the disanalogy: the same is plausibly true for low-level properties as well. Consider colour. Suppose one has a properly functioning visual system, and has an experience as of a red globe. One might employ the same technique as above and claim that this experience is accurate just in case there is some object, here and now, that instantiates the property 'being red'. There must really be, as we sometimes say, some red globe in one's visual field. So, the property REDNESS is part of the content of one's experience. But brief reflection reveals that this is too strong. First, notice how it depends upon some form of colour realism being true. If all forms of colour realism should turn out false, then colour perception is systematically inaccurate: there isn't really a red thing in one's visual field, ever. Perhaps this is unsurprising to some philosophers of colour (perhaps it is even 
the makings of a transcendental argument that some realists might employ). But it suggests that something has gone wrong with the theory if accuracy of basic forms of perception is beholden to accuracy of ontological theorizing. Second, ontological theories to one side, this specification of content still renders much of experience inaccurate. Suppose in having the experience as of a red globe, with the content specified as per above, one in fact is not in the presence of a red globe. Instead, one is in the presence of a white globe, perfectly illuminated by hidden red lighting so as to have the appearance as of a red globe. In this circumstance, the object of perception (the globe) does not instantiate the property 'being red' but only appears red (to any normal perceiver). Indeed, as described, no single object here and now instantiates 'being red'. But as specified, the experience represents the object (the globe) as instantiating the property 'being red'. Accordingly, the experience is inaccurate. This is an odd result. Situations like these are entirely ordinary and one has, by hypothesis, a normally functioning visual system. Extended, this would render a great deal of perfectly normal, appearance-tracking perception, inaccurate.

The conclusion should be that perceptual representation of high-level or kind properties and perceptual representation of low-level properties are analogous (instead of disanalogous) with respect to how properties figure into content. In both cases, there is a risk concerning the fineness of grain in identifying accuracy conditions (and thus content). Whether an experience has a reddish character or a duck-ish character, if for accuracy of the experience it is required that the properties ('being red' or 'being a duck') be, strictly speaking, instantiated here and now, then inaccuracy among normal perceivers is rampant. In other words, by parity of reasoning, if the low-level theorist maintains that the property 'being a duck' is unnecessary to adequately capture the content of the experience type with the 'being organized like a duck' character (since it would render inaccurate many relevant experiences, including the experience of the duck/rabbit), then one should maintain the same for standard low-level experiences, including colour experiences. Comparing the results of this line of reasoning to the case of aesthetic properties suggests some more surprising conclusions.

Suppose for the moment that some objectivism about all three "types" of property is true-basic properties like colour, kind properties like 'being a duck', aesthetic properties like 'being dynamic' are all real in at least the minimal sense that ascription of each type is truth-evaluable. Consideration of aesthetic properties is instructive in this context. Supposing an expert perceiver (perhaps a visual artist), perception provides full access to aesthetic properties. Aesthetic properties (or again, at least many of them) are something approaching pure appearance 
properties. ${ }^{29}$ And so a suitably trained perceiver can ascribe and identify aesthetic properties with high frequency just on the basis of her experiences. This is because there is no hidden nature or essence to a property like 'being graceful' or 'being impressionist', and this contrasts with both kind properties and basic colour properties. Glossing over a vast amount of theoretical detail and controversy, what makes something an instance of the natural kind DUCK is some underlying structure, not detectable by perception alone. And so there are many circumstances where uncertainty or mistakes about duck-identification will not be correctable by perception alone. ${ }^{30}$ Put in terms of expertise, there is no relevant expert perceiver (say an ornithologist) who, knowing all there is to know about ducks, will not have the organized-like-a-duck experience in the presence of, say, some hereto unencountered duck-looking non-duck creature from a faraway land. Correcting mis-ascription in this case requires more than what even expert perception can provide. And note, crucially, that this correction will correct judgment but will not correct perception: the expert still has an experience (of the non-duck) characterized as 'being organized like a duck'. A similar story would be told for artefactual kinds, where here the essence might involve some conventional function or role. And finally, the same is true for colour properties. Assuming again some expert perceiver (perhaps an expert colorist), there are many perceptual circumstances where vision will present the world as containing an object with some coloured appearance, but where that object in fact lacks whatever underlying (nonperceptible) features are required for an object to really be that colour. One can, at the cognitive level, correct any such-misidentification by further investigation (perhaps manipulating the environment by checking the light, or asking about the light, etc), but this does nothing to correct, in any relevant sense, the experience. The white globe in red light will still appear red to the expert, even after she knows it is not really red.

\footnotetext{
${ }^{29}$ Note that this is compatible with variation in capacity for recognizing these appearances. So, we can train ourselves, and sometimes may need to. We can better recognize appearances by repeated experience or learning of some kind. As discussed in III below, this could be achieved by a variety of cognitive mechanisms.

${ }^{30}$ For simplicity, this discussion (like all of the discussion of perception of kind properties) proceeds on the assumption that some kind of realism about kinds is true and, moreover, that we are liberal in our kind taxonomy. Thus, ducks and pine trees are natural kinds. However, the discussion could be adjusted in various ways without undermining the argument. For example, if one is a conventionalist about kinds, then the conventions (rather than some underlying microstructure) are what cannot be perceived. Or, perhaps one does not think that things like ducks or pines are kinds, but instead are species (understood as individuals or classes or sets). Here again, there will be criteria for being a member of a species that exhaust the perceptible, macrostructural features of any perceived biological thing, for example, the capacity to successfully interbreed or the sharing of a common ancestry. Indeed, this was a point anticipated even by Darwin in The Origin of Species, “... adaptive characters, although of the utmost importance to the welfare of the being, are almost valueless to the systematist. For animals belonging to two most distinct lines of descent, may readily become adapted to similar conditions, and thus assume a close external resemblance; but such resemblances will not reveal - will rather tend to conceal their blood-relationship to their proper lines of descent."
} 
The important difference between aesthetic properties vs. kind and colour properties is that sensory perception can (often) provide full access to the first but to neither of the second. Again, put somewhat crudely, aesthetic properties are something approaching pure appearance properties (exhausted by organizational gestalts); kind and colour properties are not (for reasons given just above). One might then reason that it follows that perception of the second will just more often be in error, while perception of the first is rarely in error. But this locates the difference in the wrong place, and saddles both low-level and putative high-level perception with too much inaccuracy. Instead, one should locate the difference in the world: in the way that these various properties are, or at least are theorized by our best methods of taxonomy. Accordingly, there will be differences in cognition of or judgment about or empirical investigation of these broad types of properties (i.e. how they are ascribed and identified), but there need be no general difference in how accurately they are perceptually experienced.

Here then is a radical lesson, cautiously drawn from the attempted disanalogy in the rejoinder above. Perhaps properties the natures of which are not exhausted by appearance should never figure in the contents of experience. This is clearest with kind properties. Natural kinds have essential structures hidden to perception. Sensory perception alone cannot provide access to kind properties. And so kinds are not contents of perception, because they do not figure in the accuracy conditions for experience. But what is true for kind representation is true for colour perception: if instantiation of the property 'being red' is a condition for the accuracy of perception, then many normal visual perceivers in ordinary circumstances, will suffer inaccurate experiences. Here again there are aspects to colour properties that go beyond appearances, that go beyond what perception is able to access. We are left with the awkward result that because my experience of the red-illuminated white globe says 'that is red', it is impugned as inaccurate. This holds a well-functioning visual system accountable to something that it cannot access because of the nature (or theorized nature) of the relevant properties. ${ }^{31}$

Instead, we should include in the content of perceptual experience only those properties or aspects of properties that are accessible to perception. Both kind and colour properties have characteristic appearances. That is, for most kinds and most basic properties, there is a typical feature or aspect (or cluster of aspects) that, by normal subjects, is detectable by perception

\footnotetext{
31 As an anonymous referee notes, this line of reasoning would seem to commit to a claim (of some strength) that phenomenal content (or character) determines perceptual content, and claims of this kind are controversial. This is another way in which the radical lesson drawn is a cautious one. To fully support the lesson (which is simply not the central aim or intention of this paper), then, one would need to provide a thorough analysis of the relation between sensory phenomenology and perceptual content.
} 
alone. The aspect 'being organized like a duck' is such an example. These aspects of the relevant properties are more appropriate candidates for contents qua accuracy conditions. Note what follows. In one respect, perception of aesthetic properties, kind properties, and colour properties are on a par, since in all cases those experiences are about the typical appearance aspects of said properties. In another related respect, only perception of an aesthetic property is perception of the property, since there is nothing to an aesthetic property beyond some complex organizational gestalt. But one never, strictly speaking, perceptually represents kind or colour properties.

All of this is to think of the accuracy of perception as appearance-aptness but not as truth-aptness. The latter is often assumed, but this may smuggle in considerations better reserved for doxastic cognitive states. To count as knowledge, beliefs must be true and justified. And so, because truth is the aim of belief (getting all of the facts we might say), it is appropriate that accuracy of belief qua representation is understood accordingly. A belief of the white globe illuminated in red light that it is red fails at its aim, and is accordingly inaccurate. What is different is that belief can, in this circumstance, still achieve its aim. Investigation may be required, but belief can be corrected according to its aim. So when it fails, it is not impugned for something it cannot do. This contrasts with (visual) perception. Vision cannot do any better than it does in this circumstance: even upon receipt of information about the lighting in the room, one will still experience the white globe as red. And so it shouldn't, in this case, be impugned as inaccurate. Including the property 'being red' in the accuracy conditions, and therefore content, of the perceptual experience delivers this very verdict of inaccuracy. So, properties of this kind, as well as kind properties, by contrast with aesthetic properties, should not be included in the specification of perceptual contents. ${ }^{32}$

\section{2.c: Concluding the argument: Rich perceptual content of aesthetic properties}

There are less revisionary (or at least different) ways to conclude from this analysis. So one might grant that kind properties are not (or are rarely) contents of experience, while colours and more basic properties are. One possible motivation here is the defensible view that colours just are appearance properties, by contrast to most kind properties. ${ }^{33}$ Or, one might maintain that

\footnotetext{
32 Siewert, forthcoming, suggests worries about inclusion of kind properties as such in perceptual contents, see C. Siewert, 'Respecting Appearances', in The Oxford Handbook of Contemporary Phenomenology, Ed. D. Zahavi, (Oxford, Oxford University Press, Forthcoming). Another worry looming in the vicinity here, in response to the hypothetical rejoinder, is to conclude that there is a problem not with inclusion of properties in contents but, more fundamentally, with specifying perceptual contents by specifying accuracy conditions.

33 At least with respect to perceptual representation of basic properties like colour, a related proposal is found in S. Shoemaker, 'Self-Knowledge and "Inner Sense"-Lecture III: The Phenomenal Character of Experience', Philosophy and Phenomenological Research 54 (1994), 291-314.
} 
inclusion of a property in the accuracy conditions of experience does not entail that the properties actually be instantiated in the perceiver's environment. It is only required that some member of a cluster of relevant properties (some of them perhaps gerrymandered) is instantiated, where the cluster involves the range of stimuli that typically cause the relevant broadly typed experience in normal perceivers. Thus the experience with the character 'being organized like a duck' is accurate just in case the perceiver is in the presence of a living, breathing duck or a duck decoy or a duck depiction or... This kind of proposal might be further defended if coupled with a liberal, but surely revisionary, theory of kind properties, where any instance of one of the disjuncts from the list just given is an instantiation of 'being a duck'. The important point for the proponent of AP is that, no matter one's choice about these more general matters, the work has already been done to defend the claim that aesthetic properties are admissible contents of experience.

The argument from seeing-as, briefly applied to the aesthetic contrast cases once more, ran as follows. Explanations of the contrast in strictly non-phenomenal ways or in strictly cognitivephenomenal ways are both (comparatively) implausible. The best move for the critic of AP then is to grant the phenomenal difference, but explain it in terms of differing judgments accompanied by a sensory-phenomenal difference. The sensory-phenomenal difference is not plausibly one in perceived colours or edges or other basic features; it is most plausibly a difference in the overall perceptual organization of those basic features. The impressionist spotter enjoys an experience of an organizational gestalt typical of impressionist works. And finally, to come this far is to grant rich perceptual content. An experience of this type represents more than basic colours and edges and shapes; it represents a kind of organization of features, distinctive of impressionism.

A return to the questions posed above (II.2), helps to further show that this all implies rich perceptual content, and motivation for AP. Those questions concerned what is needed to specify accuracy conditions (and so content) for perceptual experience. They were, (1) Are basic properties sufficient (so, for vision: colour, edges, shape, motion, location)? (2) Are natural or artefactual kind properties needed? The argument from seeing-as suggests a clear 'no' to question (1). The relevant part of the argument implies that specification of the contents of the contrasting aesthetic experiences (or the contrasting seeing-as a duck and seeing-as a rabbit experiences) just in terms of colours, shapes, and edges, would indicate no contrast, and so clearly not explain the apparent contrast. What is needed is specification of relevant 
organizational gestalts. But note also that, when the emphasis is on the aesthetic cases, the answer to (2) doesn't matter. Put most strongly, suppose one accepts one version of the radical lesson provided in II.2 above, that kind properties are not contents of perceptual experience. Then, trivially, one would have to answer 'no' to question (2). In effect, one can have rich perceptual content without perceptual representation of kind properties. Perception of aesthetic properties involves rich perceptual content.

\section{Further motivating AP: Mechanisms for explanation}

Additional support for AP can be adduced by noting the various cognitive mechanisms or processes that might underlie, and therefore explain, apparent instances of rich perceptual experience of aesthetic properties. Here, the proponent of AP enjoys a great deal of explanatory freedom.

Candidate mechanism 1 is cognitive penetration of perception. A number of theorists have argued that cognitive states may influence, in a relatively direct way, perceptual experience. As it is often put in the literature, two perceivers with similarly well-functioning sensory organs and attending to the same object and in the same viewing conditions, may have phenomenally distinct sensory experiences as a consequence of differences in their background beliefs. Theorists who argue that perception can be cognitively affected in this way appeal to a variety of empirical research. ${ }^{34}$ Much of the research suggests a cognitive effect on some low-level feature of conscious experience. In this respect, one might worry that the putative empirical evidence for

\footnotetext{
${ }^{34}$ Some examples of empirical research recently discussed include J.S. Bruner and C.C. Goodman., 'Value and need as organizing factors in perception.' Journal of Abnormal and Social Psychology 42 (1947), 33-44; J.S. Bruner and L. Postman 'Symbolic value as an organizing factor in perception', Journal of Social Psychology 27 (1948), 203-208. J.L. Delk and L. Fillenbaum, 'Differences in Perceived Colour as a Function of Characteristic Color.' The American Journal of Psychology 78 (1965), 290-93. T. Hansen, M. Olkkonen, S. Walter, \& K.R. Gegenfurtner, 'Memory modulates color appearance.' Nature Neuroscience 9 (2006), 1367-8; M. Olkkonen, T.. Hansen, and K.R. Gegenfurtner, 'Colour appearance of familiar objects : effects of object shape, texture and illumination changes', Journal of Vision 8 (2008), 1-16; N.R. van Ulzen, G.R. Semin, R. Oudejans, and P. Beek, 'Affective stimulus properties influence size perception and the Ebbinghaus illusion.’ Psychological Research 72 (2008), 304-310; C. Witzel, H. Valkova, T. Hansen, K. Gegenfurtner, 'Object knowledge modulates colour appearance', i-Perception 2 (2011), 13-49. Examples of recent philosophical discussion include S. Siegel 'Cognitive Penetrability and Perceptual Justification.' Nous 46 (2011), 20122 and 'The Epistemic Impact of the Etiology of Belief', Philosophical Studies 162 (2013), 697-722; F. Macpherson, 'Cognitive Penetration of Colour Experience: Rethinking the Issue in Light of an Indirect Mechanism.' Philosophy and Phenomenological Research 84 (2012), 24-62; D. Stokes, Perceiving and Desiring: A new look at the cognitive penetrability of experience.' Philosophical Studies 158 (2012), 479-92 and 'The Cognitive penetrability of perception', Philosophy Compass 8 (2013), 646-663; W. Wu, 'Visual Spatial Constancy and Modularity: Does Intention Penetrate Vision?' Philosophical Studies 165 (2013), 647-669. For a volume of new papers devoted to the topic, see A. Raftopoulos and J. Zeimbekis (Eds), Cognitive Penetrability (Oxford: Oxford University Press, 2015).
} 
cognitive penetration is of no direct import to the high-level content debate, or to the possibility of aesthetic perception. Nonetheless, the cases made for cognitive penetration, if successful, illustrate one way that background cognitive states could influence perceptual experience and should encourage new experimental investigation into the possibility of similar effects on (possible) perception of aesthetic properties and/or other high-level properties.

From a less experimental perspective, apparent cases of cognitive penetration of rich perceptual experience have been suggested and discussed in the context of the theory-ladenness of scientific observation and theorizing. For example, N.R. Hanson often described images like Figure 3 in such a way. A trained physicist, by contrast to a layperson, would experience the image distinctly, as an X-ray tube viewed from the cathode. (The reader might imagine her own, more updated, examples involving scientific imaging technologies.) Much of Hanson's discussion focuses on seeing-as and Wittgenstein's discussion thereof. In the example given, Hanson urges, it seems undeniable that the expert sees the object as an x-ray tube. And Hanson argues further, like the arguments given above, that 'seeing-as' denotes a genuine sensory phenomenon, rather than some adjunct to seeing, or some post-sensory interpretation of what has been seen. ${ }^{35}$ What's special about these alleged cases is that they involve a kind of improvement of perception, where some expertise is penetrating perceptual experience (for example, so that the technical apparatus is seen for what it is or how it can be used). The aesthetic cases might appeal to a similar mechanism, where knowledge about art penetrates perceptual experience. The expert thus sees the impressionism or gracefulness in a work that the naive can only accept by testimony. ${ }^{36}$

Candidate mechanism 2 is attention. Attentional mechanisms may be crucially involved in some instances of perception of aesthetic properties. So, with the duck/rabbit, one might think that the switch from seeing as a duck to seeing as a rabbit is enabled by attending to certain parts of the image, whereby the overall perceived organization of the image switches in the ways

\footnotetext{
35 See N.R. Hanson, Op. cit. note 15 and L. Wittgenstein, Op. cit. note 15. See also T. Kuhn, The Structure of Scientific Revolutions, (Chicago, IL: University of Chicago Press, 1962).

36 A reader familiar with these literatures might recognize a potential difference between apparent cases of cognitive penetration of basic perception (e.g. of colour or shape perception) and the cases discussed in the theory-ladenness literature, namely, that the former effects appear to be relatively synchronic, while the latter are more plausibly diachronic (e.g. where experience of a scientific image changes as one's expertise or theory changes over time). And it has often been assumed that cognitive penetration requires a relatively synchronic effect on perception. See. P. Churchland 'Perceptual Plasticity and Theoretical Neutrality: A Reply to Jerry Fodor.' Philosophy of Science 55 (1988), 167-187; J. Fodor, 'A reply to Churchland's 'Perceptual plasticity and theoretical neutrality.' Philosophy of Science 55 (1988), 188-198; D. Stokes, 'Towards a Consequentialist Understanding of Cognitive Penetration' in Cognitive Penetrability, Ed. by. A. Raftopoulos and J. Zeimbekis, (Oxford: Oxford University Press, 2015). However, this is a debate that is orthogonal to present concerns. What matters here is that perception of high-level properties is explained, and in this case by some cognitive effect (say, knowledge about art). It makes no matter to this point whether any such cases "count as" — depending upon timing of the relevant effect—cognitive penetration.
} 
described above in II.1. Similarly, one might think that a perceiver with some degree of knowledge of art attends differently to the Monet or Sisley or Pissarro and, accordingly, sees the works as impressionist. The contrast in overall perceptual organization (in the impressionist spotter vs. impressionist-naive) thus depends upon an attentional mechanism, driven by background cognitive processes. It is important to note that this could involve an intentional act of attention, but it is not obvious that it must involve this. Individuals versed in art may sometimes deliberately invoke learned methods of attending to works; but it is also probable that they have internalized some such methods. So while they know how to look they need not always think about how to look. There is a variety of research that supports this hypothesis. For example, a number of studies have suggested that saccadic eye-movement patterns vary between artistically trained versus artistically untrained perceivers. In one study, artists attend more to structural and formal features, while the untrained subjects attend more to human faces and individual objects. Artists also perform better in recall of those same pictorial features. ${ }^{37}$ And there is general evidence that attentional selection mechanisms not intentionally used by the agent affect perceptual performance. Moores et al 2003 found that object representations and associated cognitive representations strongly influence attentional selection and, in turn, perception in visual search tasks. For example, in an explicit visual search task for a MOTORBIKE image, subjects would more quickly recognize (and better recall post-stimulus presentation) an associated (but non-visually resembling) item like HELMET, with weakened recognition of unrelated distractors like LOCK or KETTLE. And in an additional experiment, the data suggests modulation of low-level (non-intentional) processing: saccadic eye movements were typically directed at images of objects associated with the explicit target in a search array. ${ }^{38,39}$

\footnotetext{
37 S. Vogt and S. Magnussen, 'Expertise in Pictorial Perception: Eye- movement Patterns and Visual Memory in Artists and Laymen', Perception 36.1 (2007), 91-100.

${ }^{38}$ E. Moores, L. Laiti, L. Chelazzi, ‘Associative knowledge controls deployment of visual selective attention' Nature Neuroscience 6 (2003), 182-89. See also M.G. Stokes, K. Atherton, E.Z. Patai, A.C. Nobre, 'Long-term memory prepares neural activity for perception' Proceedings of the National Academy of the Sciences 109 (2012), 360-67; A. Gazzaley, and A. Nobre, 'Top-down modulation: bridging selective attention and working memory' Trends in Cognitive Science 16 (2012), 129-35.

39 Those familiar with the cognitive penetration literature may recognize this as a point of debate. Attentionmediated cognitive effects on perception have often been interpreted as non-instances of cognitive penetration. Indeed, Jerry Fodor invokes this rejoinder with respect to the duck/rabbit and other ambiguous figures, arguing that perception in these cases simply involves an agent knowing how to act (attentively) so as to effect a distinct experience (say, seeing the image as a duck). Fodor may be right about this but, again, it is important to note that attention plausibly does not always take the form of an agent-driven act, and so the question is whether non-agential selection mechanisms can be caused by cognitive processes and in turn affect perception. In any case, here again the debate about (attention and) cognitive penetration is irrelevant. If seeing-as and recognizing aesthetic properties are (sometimes) to be understood as argued above, then cognitive penetration or not, perception sometimes enjoys rich content. And in the case of seeing a work as dynamic or impressionist, this rich perceptual experience may be the causal result of attentional mechanisms. The artist, for example, looks (intentionally or not) differently and so sees differently. See Fodor 1988, Op. cit. note 36. See also Stokes 2014, Op. cit. note 12 and 'Attention and the cognitive penetrability of perception (unpublished ms); Wu 2013, Op. cit. note 34; C. Mole, 'Attention-mediated cognitive
} 
Candidate mechanism 3 is perceptual learning. One standard way to characterize perceptual learning is as involving some sensory change to one's uptake of a stimulus type by virtue of repeated exposure to tokens of that type. The phenomenon involves learning insofar as the sensory change is typically performance-enhancing, for example, involving an improved capacity for discriminating instances of that stimulus type. ${ }^{40}$ Some theorists take this to be a perceptual achievement that can occur in the absence of semantic or cognitive learning. Thus one might learn to more reliably distinguish closely resembling shades of red without any sophisticated concepts for those same shades. Understood this way, perceptual learning is not the kind of mechanism that will factor into any relevant explanation which alleges a cognitive effect on perception. But here again the proponent of AP enjoys more flexibility in explanation. It is no doubt true that some alleged instances of perception of aesthetic properties are better explained by some kind of cognitive learning (perhaps in terms of cognitive penetration and/or attention as per above). But it is plausible, given that some aesthetic properties are exhausted just by appearance, that perceptual recognition thereof could involve just perceptual learning. So, one can imagine a perceiver who repeatedly views ballet performances, while remaining ignorant of any relevant theories, terms, or sophisticated concepts about the art form (or, to strengthen the case, any art form or theory). It is still plausible that, as exposure is increased, the perceiver may acquire a capacity for discriminating graceful performance from non-graceful performance. She would have thereby acquired a capacity for seeing certain actions as graceful, as organized in the ways typical of 'being graceful', but without possessing the relevant terms. This would be rich perceptual experience of an aesthetic property, enabled by perceptual learning.

Finally, if used in the service of explaining AP, and the arguments given above, these three types of mechanism are non-exclusive. Some instances of rich perception of aesthetic properties may involve cognitive penetration; others may involve perceptual learning. There is no feature of AP, nor the arguments given for it here, that implies or requires a singular underlying mechanism for each instance of the phenomenon. Moreover, the three types are in some cases complementary. Instances of perceptual learning, for example, very probably involve changes in attentional behaviours as the subject is repeatedly exposed to similar stimulus patterns. Accordingly, a full

penetration', in Cognitive Penetrability, Ed. by. A. Raftopoulos and J. Zeimbekis, (Oxford: Oxford University Press, 2015).

40 Two classic discussions of perceptual learning are JJ. Gibson and E.J. Gibson, 'Perceptual Learning: Differentiation or enrichment?’ Psychological Review 62 (1955), 32-41 and E.J. Gibson, Principles of Perceptual Learning and Development (New York: Appleton-Century-Crofts, 1969). For a more recent empirical review, see R.L. Goldstone, 'Perceptual learning', Annual Review of Psychology 49 (1988), 585-612. 
theory of aesthetic perception would best explore each of these cognitive mechanisms, which could in turn be used to further buttress the case for AP.

\section{A conclusion and a speculation}

The argument from seeing-as was first applied to ambiguous figures. When one sees the duck/rabbit figure as a duck, one enjoys an experience best phenomenally characterized as 'being organized like a duck'. The argument concluded that there is no way to explain the contrast between this experience and the seeing-as a rabbit experience without admitting perceptual discrimination of an overall organization, namely, that typical of a duck. That content is not specified just in terms of low-level properties. But it also need not, and perhaps should not, be specified in terms of the natural kind 'being a duck'. The argument is even more compelling when applied to aesthetic properties. There the claim was that in cases of aesthetic reaction, explaining phenomenal differences requires invoking an organizational gestalt: when one sees a work as impressionist, one perceives some overall impressionist gestalt. But, with aesthetic properties like these, to perceptually experience the relevant gestalt just is to perceptually experience the property. Therefore, aesthetic properties can figure in the contents of perceptual experience.

At the very least this argument—coupled with the rich array of cognitive mechanisms that might (non-exclusively) explain contrasts in aesthetic reaction-shifts the burden of proof to the sceptic of AP. And if AP can be defended against the other two worries-ontological and hedonic - then a traditional intuition will be vindicated: the aesthetic is a perceptual phenomenon. ${ }^{41}$

Finally, to end on a speculative note. Consideration of aesthetic cases-both in terms of aesthetic properties and ambiguous pictorial representation-sheds novel light on general philosophy of perception. If the above analysis is successful, aesthetic properties are sometimes perceptually represented. And seeing-as, at least when viewing pictorial representations, often involves perceptual recognition of the organizational gestalts typical of kinds of things. This is rich perceptual experience. And it is rich perceptual experience without perceptual representation of kind properties. The general proposal, only partly defended in II.2, was that kind properties may be inappropriate contents for experience, not because sensory perception fails in any sense to represent them but because kinds, being what they are (or are theorized to be), are not the

${ }^{41}$ At least some of the time, the unexciting qualification should be. 
sorts of things to which perception can be sensitive. What it can represent, and more or less accurately, is the way basic features of an object are organized. This is the phenomenal character that pops out when one shifts from seeing-as a duck to seeing-as a rabbit, and perhaps it is what pops out when one learns to recognize pine trees.

This would be neither a retreat for the rich content theorist, nor a trivial revision of the thesis. These experiences are rich not just in the mere contrastive sense that they represent "more than" the basic features of colour, shape, and so on. They are rich in ways that matter to various theoretical agendas traceable to the first explicit discussions of rich content and kinds: to theories of cognitive architecture, psychopathology, epistemology. Focusing just on epistemology, there are two sides to the coin. First, if experience is potentially rich, then we can acquire more information by perceiving the world, and so some sceptical worries may be less motivated. Second, if an experience is rich, it will serve as a justificatory basis for fewer belief contents. ${ }^{42}$ Specifying rich contents as involving organizational gestalts typical of kinds, but not kind properties themselves, still implies both consequences. Take Siegel's relevant example: Suppose I know what peaches are, and I am in the visual presence of a peach. Generalizing the suggestion given in this paper, this experience gives me more than mere colours, shapes, and location, but also a phenomenally salient organization of these features typical of a kind of thing. If the kind 'being a peach' is not included in the content of the experience, I don't get immediate justification from my experience for the belief that 'that is a peach'. Instead what I get, at most, is some link between perceptual content involving the organized-like-a-peach gestalt and the resultant belief, where this link involves various beliefs and perceptual memories about instances of the kind and their typical appearances. The beliefs that this experience can justify are accordingly restricted, given the learned link between gestalt and kind. By contrast with the peach-naive, I'd be justified in a belief about the presence of a peach but not about the presence of, say, an orange, bouncy ball. On this view, perception does not do everything for me, but it does do what it can.

\section{Acknowledgements}

Special thanks to Anna Bergqvist and Robert Cowan, and an anonymous referee, for helpful feedback and suggestions. Versions of this paper were given at the Conference on Perception and the Arts, Institute of Philosophy, London, and at the Impure Perception Conference at the Berlin School of Mind and Brain, Berlin. Special thanks to the organizers of these events: Bence

\footnotetext{
${ }^{42}$ See Siegel, Op. Cit. Note 3.
} 
Nanay, and Joerg Fingerhut, Lara Pourabdolrahim, and Jesse Prinz, and to the participants at these events for critical feedback.

Figure 1
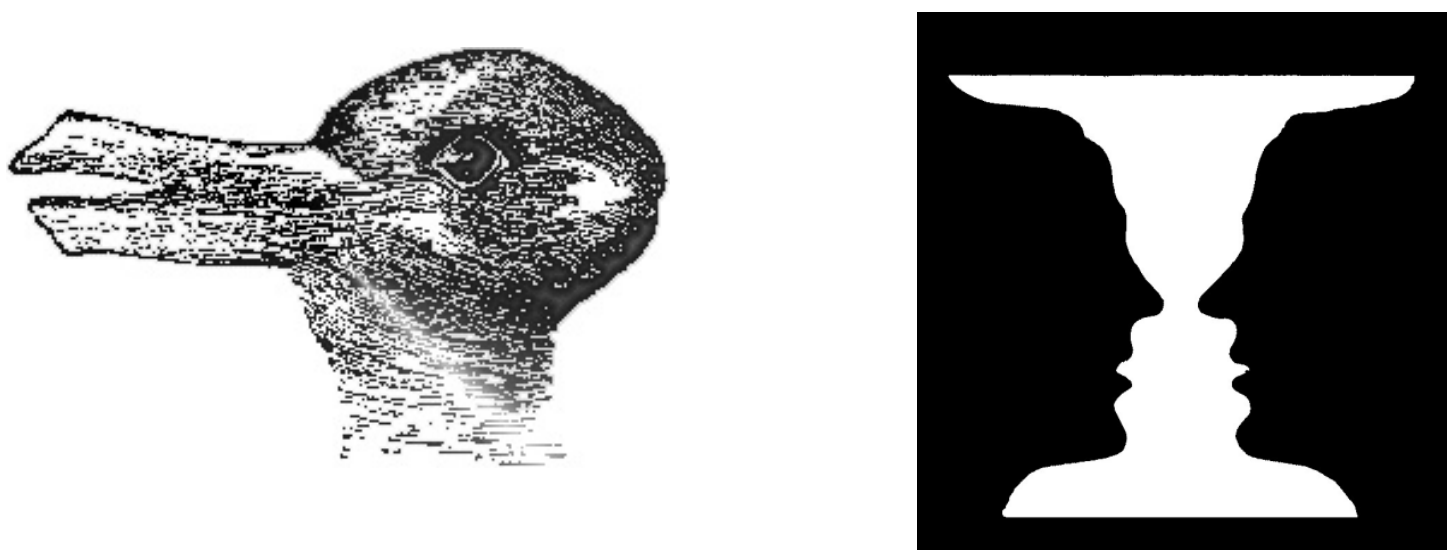

Figure 2

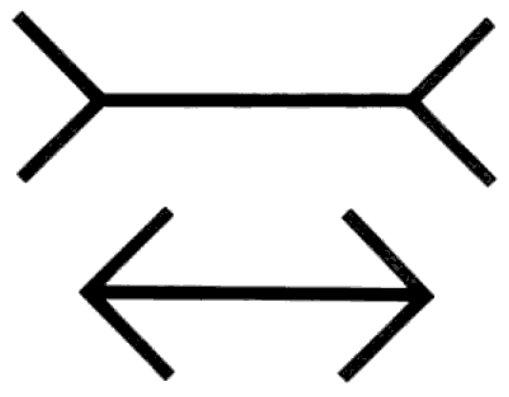


Figure 3

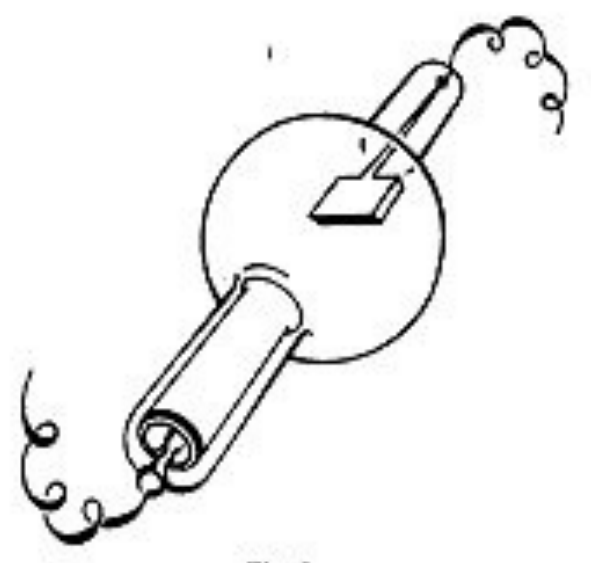

Fig. 8 